OPEN ACCESS

Edited by:

J. Arturo García-Horsman, University of Helsinki, Finland

Reviewed by:

Luca Murru,

Consiglio Nazionale delle Ricerche

(CNR), Italy

Brian R. Christie,

University of Victoria, Canada

*Correspondence:

Gert Lubec

gert.lubec@/ubeclab.com

Received: 25 September 2017 Accepted: 09 November 2017

Published: 23 November 2017

Citation:

Smidak R, Sialana FJ, Kristofova M, Stojanovic T, Rajcic D, Malikovic J,

Feyissa $D D$, Korz $V$, Hoeger $H$, Wackerlig J, Mechtcheriakova $D$ and

Lubec G (2017) Reduced Levels of the Synaptic Functional Regulator FMRP in Dentate Gyrus of the Aging

Sprague-Dawley Rat.

Front. Aging Neurosci. 9:384 doi: 10.3389/fnagi.2017.00384

\title{
Reduced Levels of the Synaptic Functional Regulator FMRP in Dentate Gyrus of the Aging Sprague-Dawley Rat
}

\begin{abstract}
Roman Smidak', Fernando J. Sialana', Martina Kristofova', Tamara Stojanovic', Dragana Rajcic', Jovana Malikovic' ${ }^{1}$, Daniel D. Feyissa', Volker Korz², Harald Hoeger², Judit Wackerlig', Diana Mechtcheriakova ${ }^{3}$ and Gert Lubec ${ }^{1,4 *}$
\end{abstract}

${ }^{1}$ Department of Pharmaceutical Chemistry, Faculty of Life Sciences, University of Vienna, Vienna, Austria, ${ }^{2}$ Core Unit of Biomedical Research, Division of Laboratory Animal Science and Genetics, Medical University of Vienna, Vienna, Austria, ${ }^{3}$ Department of Pathophysiology and Allergy Research, Medical University of Vienna, Vienna, Austria, ${ }^{4}$ Neuroproteomics, Paracelsus Private Medical University, Salzburg, Austria

Fragile $X$ mental retardation protein (FMRP) encoded by Fragile $X$ mental retardation 1 (FMR1) gene is a RNA-binding regulator of mRNA translation, transport and stability with multiple targets responsible for proper synaptic function. Epigenetic silencing of FMR1 gene expression leads to the development of Fragile $X$ syndrome (FXS) that is characterized by intellectual disability and other behavioral problems including autism. In the rat FXS model, the lack of FMRP caused a deficit in hippocampal-dependent memory. However, the hippocampal changes of FMRP in aging rats are not fully elucidated. The current study addresses the changes in FMRP levels in dentate gyrus (DG) from young (17 weeks) and aging (22 months) Sprague - Dawley rats. The aging animal group showed significant decline in spatial reference memory. Protein samples from five rats per each group were analyzed by quantitative proteomic analysis resulting in 153 significantly changed proteins. FMRP showed significant reduction in aging animals which was confirmed by immunoblotting and immunofluorescence microscopy. Furthermore, bioinformatic analysis of the differential protein dataset revealed several functionally related protein groups with individual interactions with FMRP. These include high representation of the RNA translation and processing machinery connected to FMRP and other RNA-binding regulators including CAPRIN1, the members of Pumilio (PUM) and CUG-BP, Elav-like (CELF) family, and YTH N(6)-methyladenosine RNAbinding proteins (YTHDF). The results of the current study point to the important role of FMRP and regulation of RNA processing in the rat DG and memory decline during the aging process.

\footnotetext{
Keywords: Fragile $\mathrm{X}$ syndrome, Fragile $\mathrm{X}$ mental retardation protein, brain aging, dentate gyrus, proteomics, RNA-binding protein
}

\section{INTRODUCTION}

The Fragile X mental retardation protein (FMRP) is a RNA-binding regulator encoded by Fragile $\mathrm{X}$ mental retardation 1 (FMR1) gene. This protein is ubiquitously produced in mammalian tissues with high levels in brain (Tamanini et al., 1997), and localized predominantly in cytosolic light and heavy membranes, and nucleus (Taha et al., 2014). It contains two K-homology domains 
(KH1 and KH2) and a C-terminal RGG box, involved in RNAbinding, together with nuclear localization and nuclear export signal (Adinolfi et al., 1999; Valverde et al., 2007). These structural features suggest the molecular functions of FMRP which have been associated with mRNA stability (Zalfa et al., 2007), nuclear cytoplasmic shuttling of mRNA (Eberhart et al., 1996) and repression of mRNA translation (Jin and Warren, 2000; Li et al., 2001; Kalidas and Smith, 2003). The total number of FMRP functions in vivo is not known and a multitude of FMRP targets was proposed based on large-scale analyses (Ascano et al., 2012; Ouwenga and Dougherty, 2015).

Fragile X mental retardation protein has been implicated as a regulator of synaptic plasticity and thereby learning and memory (reviewed in Bostrom et al., 2016). The functions of FMRP in nervous system have been related to the control of synaptic protein synthesis in response to neurotransmitter activation and affecting the formation of axonal and dendritic structures (Feng et al., 1997; Greenough et al., 2001; Weiler et al., 2004; Zimmer et al., 2017). The FMRP-mediated regulation of mRNA translation has been also directly linked to neurotransmitter receptor systems involved in synaptic plasticity. As an example, it was shown that the activation of metabotropic glutamate receptors (mGluR) affects FMRP localization in dendrites and synapses (Antar et al., 2004), FMRP deficiency leads to excessive mGluR5- and local protein synthesis-dependent internalization of AMPA receptors (Nakamoto et al., 2007) and that the expression of the NMDA receptor subunit NR2A is regulated by FMRP-associated microRNAs (Edbauer et al., 2010).

Impairment of FMR1 gene expression due to amplification of CGG repeat in its $5^{\prime}$-UTR and hypermethylation leads to several syndromes. The full mutation allele ( $>200$ CGG repeats) results in lack of FMRP and development of Fragile X syndrome (FXS) that is characterized by cognitive disabilities and behavioral disorders (Wang et al., 2012). The mouse and rat FMR1 knockout $(\mathrm{KO})$ models have been extensively used to study behavioral deficits, and alterations in brain physiology and development associated with FXR (Till et al., 2012, 2015). Interestingly, a recent quantitative proteomic study of FMR1 KO mouse revealed a significant overlap of a dysregulated proteome between the FXR condition and normal aging (Tang et al., 2015) and the FMRP levels were found to be decreased in aged mouse brain (Singh et al., 2007; Singh and Prasad, 2008).

The information on aging-dependent changes of FMRP in rat brain and specific brain subareas is limited. The dentate gyrus (DG) is a hippocampal subregion that plays a critical role in information processing including spatial memory (Jonas and Lisman, 2014; Bott et al., 2016) and the aging-related functional alterations in this region were previously described for rats, mice, and other mammalian models (Lister and Barnes, 2009). The results obtained from FMR1 KO mouse models indicate an important function of FMRP in physiology, structure and connectivity of DG, and the related behavior (Bostrom et al., 2016; Lai et al., 2016; Scharkowski et al., 2017). Previous studies on rats showed deficits in hippocampal-dependent memory in a FMR1 KO model (Till et al., 2015) and behavior-induced changes of FMRP level in DG (Irwin et al., 2005). However, validated information on FMRP levels in DG in rat during normal aging is missing. In the current study, FMRP levels in DG were compared between the young (17 weeks) and aging (22 months) SpragueDawley rats. In the initial hole-board test the aging group showed a significant decline in spatial reference memory. Protein samples of DG from five rats per each group were then analyzed by quantitative proteomic analysis resulting in 153 significantly changed proteins. FMRP showed significant reduction in aging animals which was confirmed by immunoblotting and immunofluorescence microscopy. Furthermore, bioinformatic analysis of the differential protein dataset revealed several functionally related protein groups with individual interactions with FMRP. These included high representation of RNA translation and processing machinery connected to FMRP and other RNA-binding regulators. The results of the current study point to an important role of FMRP and the regulation of RNA processing in the rat DG related to memory decline during the aging process.

\section{MATERIALS AND METHODS}

\section{Animals}

Animals were bred and maintained in the Core Unit of Biomedical Research, Division of Laboratory Animal Science and Genetics, Medical University of Vienna. The animals lived in a separate experimental room 1 week before and throughout the experiment. Rats were housed individually in standard Makrolon cages filled with autoclaved woodchips (temperature: $22 \pm 2{ }^{\circ} \mathrm{C}$; humidity: $55 \pm 5 \%$; $12 \mathrm{~h}$ artificial light/12 h dark cycle: light on at 7:00 am). The study was carried out in accordance the recommendation of the European Communities Council Directive of 24 November 1986 (86/609/EEC) evaluated by the ethics committee of the Medical University of Vienna, Vienna, Austria. The protocol was approved by Federal Ministry of Education, Science and Culture, Austria.

\section{Hole-Board Memory Test}

The hole-board consisted of a $1 \mathrm{~m} \times 1 \mathrm{~m}$ board made of black plastic surrounded by translucent plexiglass walls. Each side of the wall bears proximal spatial cues. Distal cues were provided by room structures visible outside the board. Four out of sixteen regularly arranged holes (diameter and depth $7 \mathrm{~cm}$ ) were baited (dustless precision pellets, $45 \mathrm{mg}$, Bioserv ${ }^{\circledR}$ ). The pattern of baited holes remained the same during the entire test. In order to avoid olfactory orientation pellets were also present in an area below the board. Rats were familiarized to the experimenter through $10 \mathrm{~min}$ handling sessions per day for 4 days prior to the experiment, followed by 2 days of habituation to the hole-board during which the animals were allowed to explore the maze for $15 \mathrm{~min}$ each day with access to food pellets. The weight of each rat was gradually reduced by food restriction to reach $85 \%$ of its free-feeding body weight before the start of the experiment. Tap water was given ad libitum. Thereafter the rats were trained for 3 days (five trials on day 1 , four trials on day 2 and a retention trial at day 3 ). Every trial lasted for $120 \mathrm{~s}$ or until all four pellets were eaten. The apparatus was cleaned with $0.1 \%$ incidin between trials in order to remove the rat odor cues. The interval between two successive 
trials for an individual was $20 \mathrm{~min}$. A camera mounted on the room ceiling recorded the performance of the rats in the maze. The visits of holes and removals of pellets were noted for each trial. In order to compare rats with similar levels of motivation, rats with less than 40 hole-visits in total over the 10 trials were excluded from the analysis. Reference memory errors were noted as the number of visits to the unbaited holes. The Reference Memory Index (RMI) was calculated using the formula: (first + revisits of baited holes)/total visits of all holes. All behavioral training/testing was performed during the light phase of the light-dark cycle. Rats were decapitated, the brains were rapidly removed and dissected on a Para Cooler (RWW Medizintechnik, Hallerndorf, Germany) at $4^{\circ} \mathrm{C}$ to obtain DG. The tissue was immediately stored at $-80^{\circ} \mathrm{C}$ until further analysis.

\section{Protein Sample Preparation}

All homogenization and centrifugation steps were carried out on ice and at $4^{\circ} \mathrm{C}$, respectively. Brain tissues were homogenized in an ice-cold homogenization buffer [10 mM HEPES, $\mathrm{pH}$ 7.5, $300 \mathrm{mM}$ sucrose, protease inhibitors (Roche)] using a Dounce homogenizer; the homogenate was centrifuged at $1000 \times g$ for $10 \mathrm{~min}$ to remove cell debris and nuclei and the supernatant was collected. The pellet was resuspended again in the homogenization buffer and centrifuged at $1000 \times g$ for $10 \mathrm{~min}$. The pooled supernatants were then centrifuged at $100000 \times g$ for 60 min to enrich membranes. The resulting membrane pellets were washed in $10 \mathrm{mM}$ HEPES, $\mathrm{pH} 7.5$, protease inhibitors (Roche) and solubilized in $50 \mathrm{mM}$ TEAB buffer (Sigma-Aldrich), $7 \mathrm{M}$ urea, $2 \mathrm{M}$ thiourea, 4\% CHAPS, $100 \mathrm{mM}$ DTT and protease inhibitors (Roche). The protein concentration was determined by Pierce ${ }^{\mathrm{TM}} 660 \mathrm{~nm}$ Protein Assay (Thermo Scientific).

\section{Quantitative LC-MS/MS Analysis}

Protein samples were digested overnight with trypsin (Promega) using filter-aided sample preparation (FASP) (Wisniewski et al., 2009) with $50 \mu \mathrm{g}$ of protein per one reaction. The tryptic peptides were desalted using reversed-phase C18 stage tips (Rappsilber et al., 2007) and reconstituted in $50 \mu \mathrm{L}$ of $50 \mathrm{mM}$ TEAB (Sigma-Aldrich, Hamburg, Germany). The samples (50 $\mu \mathrm{g}$ in $50 \mu \mathrm{L}$ ) were subsequently differentially labeled by TMT10plex ${ }^{\text {TM }}$ Isobaric Label Reagent Set (Thermo Scientific) according to the manufacturer's instructions. Using an actual peptide concentration determined by Pierce ${ }^{\mathrm{TM}}$ Quantitative Colorimetric Peptide Assay (Thermo Scientific) the samples were pooled by mixing an equal amount of labeled peptides from individual samples. The pooled sample was separated by reversed-phase liquid chromatography (RP-LC) at high $\mathrm{pH}$ as previously described (Gilar et al., 2005) resulting in 25 fractions. The peptide fractions were analyzed by liquid chromatography-tandem mass spectrometry (LC-MS/MS) with two technical replicates per sample using the LC separation procedure described previously (Smidak et al., 2016). MS analysis was performed by the Thermo Scientific ${ }^{\mathrm{TM}} \mathrm{Q}$ Exactive $^{\mathrm{TM}}$ Plus Orbitrap mass spectrometer (Thermo Scientific) in positive ion mode with full-scan MS in the range of $m / z \quad 375-1400$ at the resolution of 35,000 (at $\mathrm{m} / \mathrm{z} 200$ ). MS/MS scans were acquired at the resolution of 70,000 (m/z 200) through HCD fragmentation of 15 most intense ions at $32 \%$ normalized collision energy with a fixed mass of $100 \mathrm{~m} / \mathrm{z}$. Dynamic exclusion was enabled for $30 \mathrm{~s}$ and for unassigned, +1 and $\geq+8$ charges.

\section{Proteomic Data Analysis}

MS raw data were searched against UniProtKB Rattus norvegicus complete proteome database (UP000002494, 31569 sequences, downloaded on December 2nd, 2016) using MASCOT 2.4 (MatrixScience) through Proteome Discoverer 2.1 platform (Thermo Scientific). The search criteria were as follows: trypsin with a maximum of two missing cleavage sites; fixed modification: carbamidomethylation (C); variable modification: oxidation (M); search mode: MS/MS ion search with decoy database search included; peptide mass tolerance $\pm 10 \mathrm{ppm}$; MS/MS mass tolerance $\pm 0.02 \mathrm{Da}$; and protein and peptide FDR 1\%. Only proteins identified by two distinct peptides and one unique peptide were considered for the final dataset. The Reporter Ions Quantifier node in the Proteome Discoverer software was used to calculate the corresponding protein abundances based on the intensities of individual TMT reporter ion channels $(126.13-131.14 \mathrm{~m} / \mathrm{z})$ corrected for reporter ions isotopic distribution. The protein abundances were normalized by the total intensity of each reporter in the PSMs (PeptideSpectral-Matches) population. Differences in protein levels are expressed as log2-transformed normalized protein abundance ratio of aging versus young animal group evaluated by twosided $t$-test using Perseus statistical package (version 1.3.0.4). $p$-Values $<0.05$ with permutation-based FDR threshold 0.05 were used as cut-off for significance. Additional filtering for significantly changed proteins was applied based on log2 (protein ratio) threshold $>|0.3|$. Significantly changed proteins were analyzed for enrichment of GO annotations using ClueGOCytoscape platform (Bindea et al., 2009; Huntley et al., 2015) using GOA database 30.08.2017 and Benjamini-Hochberg correction (adjusted $p$-Values $<0.01$ ). The lists of most representative pathways and biological functions/diseases, and protein interaction networks were generated by Ingenuity Pathway Analysis (IPA) software (Qiagen) using right-tailed Fisher's exact test $(p<0.05)$. Functionally related protein clusters within the differential dataset were visualized by STRING v10.5 ${ }^{1}$ and MCL algorithm with confidence threshold 0.5 and inflation parameter 3 .

\section{Western Blot}

The protein samples were separated by electrophoresis in $10 \%$ SDS-PAGE gel and transferred to PVDF membranes (GE Healthcare). The western blot procedure was performed as described previously (Shanmugasundaram et al., 2017) with minor modifications. Briefly, the membrane was blocked for $1 \mathrm{~h}$ in 5\% non-fat milk, $1 x$ TBS, $0.05 \%$ Tween- 20 and probed with the corresponding primary and secondary antibodies. For detection of FMRP protein the membrane was incubated in 3\% non-fat milk, $1 x$ TBS, $0.1 \%$ Tween-20 with 1:1000 polyclonal mouse antiFMRP antibody (MAB2160, Millipore) overnight at $4^{\circ} \mathrm{C}$ followed

\footnotetext{
${ }^{1}$ https://string-db.org/
} 
by incubation for $1 \mathrm{~h}$ at room temperature in 1:10000 HRPconjugated anti-mouse secondary antibody (ab6728, Abcam). Actin as a loading control was detected by incubation in $3 \%$ nonfat dry milk, $1 \mathrm{x}$ TBS, $0.1 \%$ Tween-20 with 1:2000 anti- $\beta$-Actin antibody (4967S, Cell Signalling) overnight at $4^{\circ} \mathrm{C}$ followed by incubation for $1 \mathrm{~h}$ at room temperature in 1:10000 rabbit polyclonal antibody. The signals were quantified using ImageJ (NIH) software based on calculation of peak area and the intensity values for FMRP bands were normalized across the samples by actin control.

\section{Immunofluorescence Detection and Microscopy}

For the analysis of FMRP expression eight young and eight aging rats were deeply anesthetized with isoflurane and transcardially perfused with $4 \%$ paraformaldehyde solution in $0.1 \mathrm{M}$ sodium phosphate buffer, $\mathrm{pH}$ 7.4. Brains were gently removed and post-fixed in the same fixative solution for $20-24 \mathrm{~h}$ at $4^{\circ} \mathrm{C}$. Each brain was rinsed well with phosphate buffered saline (PBS) to remove formaldehyde and incubated in 30\% sucrose with $0.02 \% \mathrm{NaN}_{3}$ at $4^{\circ} \mathrm{C}$. Immediately prior to cutting, brains were embedded in O.C.T. Tissue-Tek (Sakura Finetek) and frozen at $-20^{\circ} \mathrm{C}$ in a cryo chamber. Tissue was sectioned at $20 \mu \mathrm{m}$ with a cryostat CryoStar NX50 (Thermo Scientific). Coronal cryo sections (Bregma 3.30-3.80 mm) were blocked in 5\% normal goat serum (NGS) (G9023, Sigma-Aldrich), 0.3\% Triton X-100 (T8787, Sigma-Aldrich), 2\% BSA (23208, Thermo Scientific) in $0.1 \mathrm{M}$ PBS 7.4 for $2 \mathrm{~h}$ at $22-24^{\circ} \mathrm{C}$ and incubated with primary antibody (mouse anti-FMRP 1:500 MAB2016, Merck) for $48 \mathrm{~h}$ at $4^{\circ} \mathrm{C}$ in $0.1 \mathrm{M}$ PBS, $1 \% \mathrm{NGS}, 0.1 \%$ Triton X-100, 0.1\% BSA with continuous stirring. After three washing steps in $0.1 \mathrm{M}$ PBS, sections were incubated with the secondary antibody (1:1000 AlexaFluor488 anti-mouse 4408S, Cell Signaling Technology) for $2 \mathrm{~h}$ at $22-24^{\circ} \mathrm{C}$ in $2 \%$ BSA. In addition, to remove lipofuscin-like autoimmunofluorescence, all sections were incubated in autoimmunofluorescence eliminator reagent (2160, Merck) for $5 \mathrm{~min}$ and then underwent six washing steps for 1 min each in $70 \%$ ethanol. DAPI staining was used to assess the gross cell morphology (D1306, Thermo Scientific). All sections were mounted with DAKO fluorescence mounting medium (Agilent Technologies) and analyzed by fluorescence microscopy. Immunoreactivity signal of FMRP in cells, processes and neutropil in the DG were acquired on a Zeiss LSM780 confocal microscope (Zeiss, Jena, Germany) using 20x magnification. The image analysis software Image $(\mathrm{NIH})$ calculated the percentage of FMRP-positive area per section by thresholding FMRP immunoreactivity above background levels. The cell body layer of DG granule cells and hilus were separately outlined as regions of interest (ROI) according to the DAPI signal in each slice. Immunofluorescence imaging and data analysis were duplicated, evaluating two brain slices per rat in total.

\section{Statistical Analysis}

Data obtained from western blot and immunofluorescence microscopy was analyzed using Microsoft Excel 2013 or Prism 5 (GraphPad Software) and the difference between animal groups was evaluated by Student's $t$-test with the significance cut-off $p<0.05$. The behavioral data was analyzed with SPSS Version 22 software $\left(\right.$ IBM $^{\circledR}$ SPSS $^{\circledR}$ Statistics) using general linear model repeated measures ANOVA for the differences over the entire training, or separately for days 1 and 2, and univariate ANOVA for the retention trial. The significance cut-off was set at $p<0.05$. The values are expressed as mean \pm SEM.

\section{RESULTS}

\section{Behavioral Testing}

Behavioral testing was performed to assess hippocampaldependent memory performance in the subarea of interest. The cohort of 18 young and 104 aging Sprague-Dawley rats were evaluated for spatial reference memory using the hole-board test. A significant effect of the entire training on memory performance ( $p=0.032$ ) was observed indicating that learning took place. Furthermore, the data showed a significant effect of the trainingage factor interaction $(p=0.001)$ when the entire training was compared. The significant group differences were observed on day $2(p=0.001)$ and at the retention test at day $3(p=0.011)$ with the group of young animals performing significantly better than aging group (Supplementary Figure S1).

\section{Quantitative Proteomics Reveals Decreased Levels of FMRP}

A quantitative MS-based approach was employed to identify proteins with significantly changed levels in the aging rats. The experimental design included two - young and aging animal groups used in the previous behavioral test, five biological replicates per group. The membrane-enriched protein samples from DG were analyzed by LC-MS as TMT10plex labeling experiments in two sets representing two technical replicates per one biological replicate, i.e., two MS runs of each peptide fraction. Quantitative data for each individual are expressed as normalized protein abundance values for the corresponding TMT channel. A total number of 5832 proteins was identified in both groups, of which 153 were showing significantly different levels $[p<0.05$, $\log 2$ (protein ratio) $>|0.3|]$ between the groups. The complete list of significantly changed proteins as well as all identified proteins in the study is provided as supporting information (Supplementary Table S1).

The FMRP was unambiguously identified in the proteomic samples as downregulated $[\log 2 \quad$ (protein abundance ratio) $=-0.39, p=0.0005$ ] in the aging group (Supplementary Table S1) which was confirmed by western blotting (Figure 1A). The aging-dependent changes for FMRP in hippocampus were further analyzed by immunoflourescence microscopy with eight young and eight aging rats of the same behaviorally tested cohort. Consistently with general reduction in FMRP levels, there was a significant decrease $(p=0.002)$ in the total FMRP immunoreactivity per DG region in aging (7.86 \pm 2.38$)$ compared to young (12.85 \pm 2.21$)$ animals (Figure 1B). Furthermore, the comparison of individual DG subareas showed a significant decrease in FMRP labeling in aging rats in both, 
A

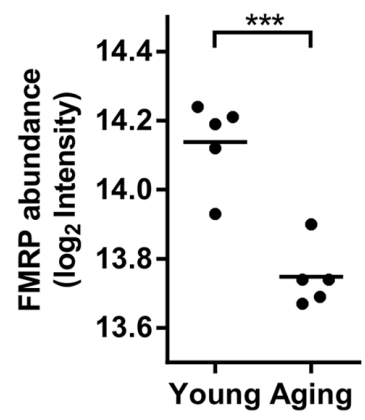

B
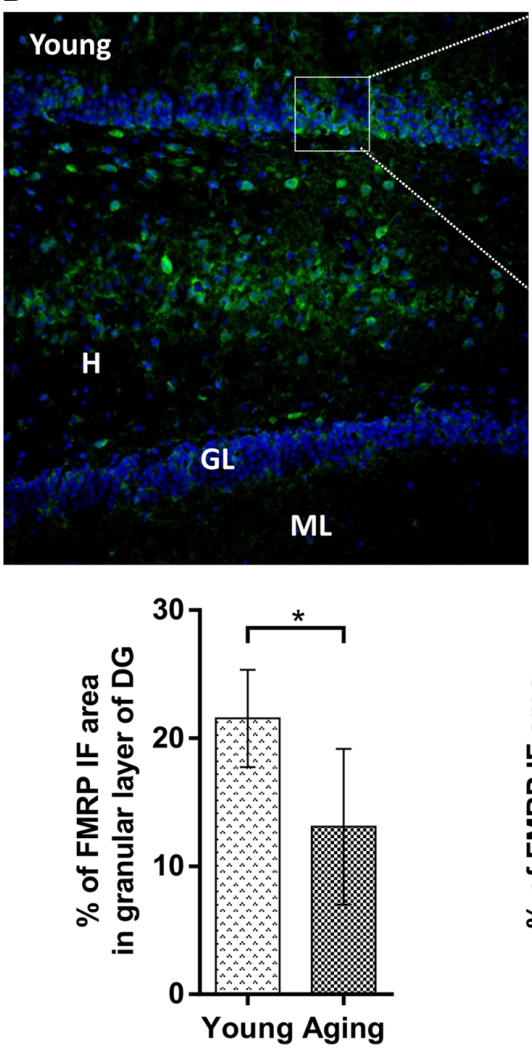

Young

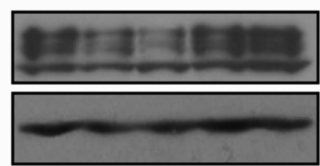

Aging
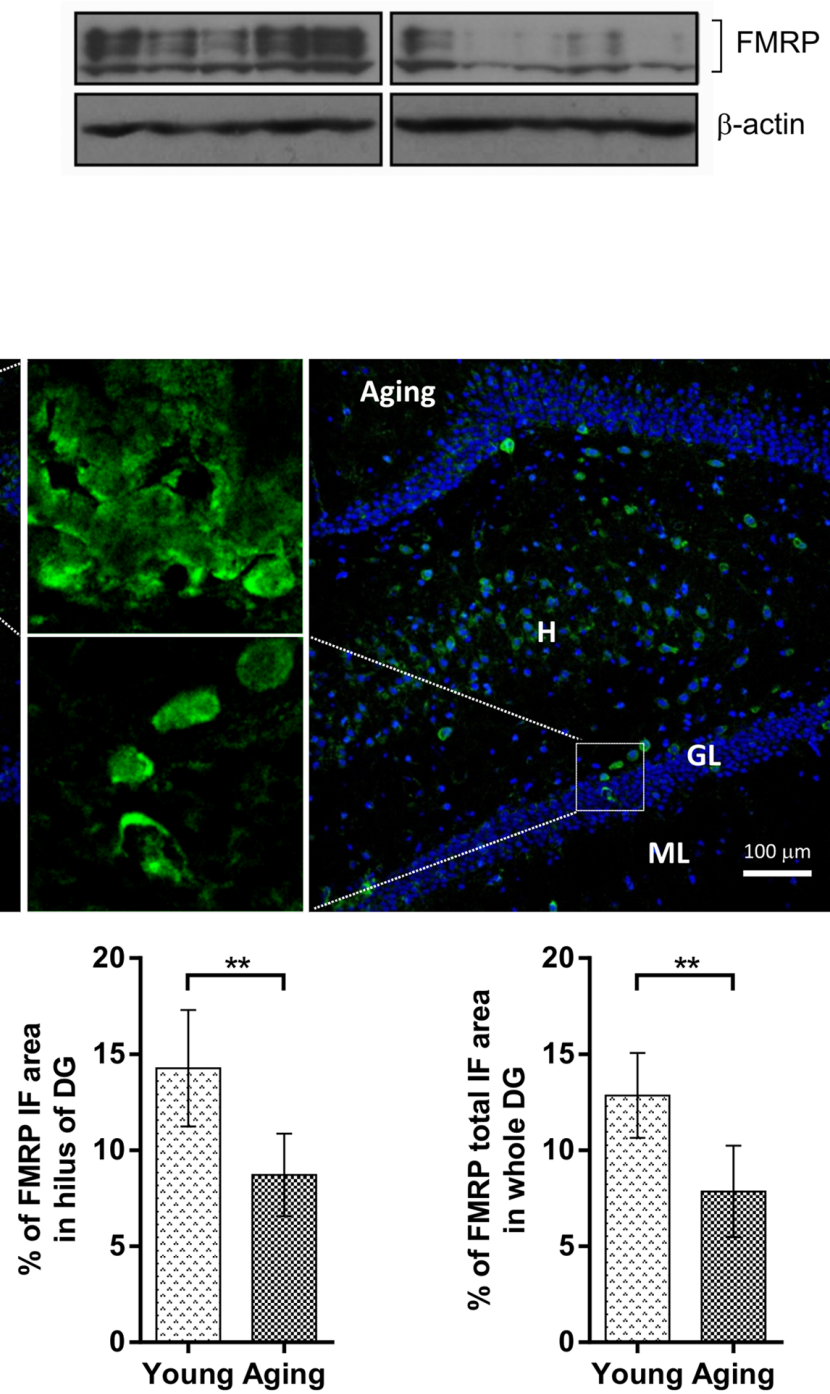

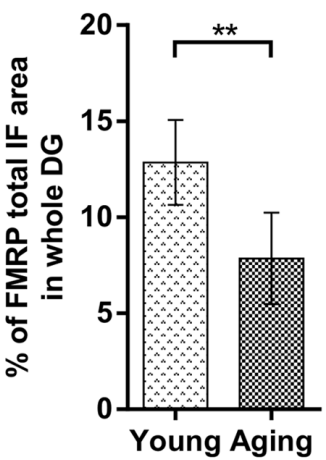

FIGURE 1 | Comparison of FMRP levels between aging and young rats based on proteomics, immunoblotting and immunofluorescence (IF) microscopy. (A) The protein abundance levels of FMRP in young and aging rats obtained from a quantitative proteomic study (left) and immunoblot analysis (right). (B) Distribution of FMRP immunofluorescence analyzed in the hippocampal subareas of young and aging rats. Fluorescence images represent $10 \times, 20 \times$, and $100 \times$ magnification of DG with FMRP labeled cells and neuropil in young and aging rats. Graphs represent percentage $(\%)$ of FMRP immunoreactivity in the granular layer (GL) and hilar (H) subareas of the DG area, as well as percentage (\%) of total FMRP immunoreactivity throughout the whole DG. The differences were evaluated using Student's $t$-test $(p<0.05)$. Values are expressed as mean \pm SEM. ${ }^{*} p<0.05,{ }^{* *} p<0.01,{ }^{* * *} p<0.001$

hilus (14.27 \pm 3.03 vs. $8.72 \pm 2.15, p=0.002)$ and granular cell layer $(21.55 \pm 3.80$ vs. $13.09 \pm 6.08, p=0.01)$.

\section{Bioinformatic Analysis of Proteomic Results}

To dissect the global proteomic changes identified in the current study functional enrichment analysis was applied to differential protein dataset. The biological processes and molecular functions that may be altered in aging animals were identified based on Gene Onthology (GO) enrichment $(p<0.01)$. The results for biological processes showed the overrepresentation of GO terms associated with RNA metabolism, regulation of RNA splicing and stability, membrane raft organization, cell junction assembly, regulation of endocytosis, and synaptic plasticity. The most significant molecular functions were related to RNA binding activity, glycosaminoglycan binding activity, structural constituents of extracellular matrix and cytoskeleton (Figure 2A). For more detailed annotation the data were submitted to IPA software (Qiagen) that infers activation states (z-score) of the representative pathways and biological 
A

\section{Biological Process \\ Biological Process}

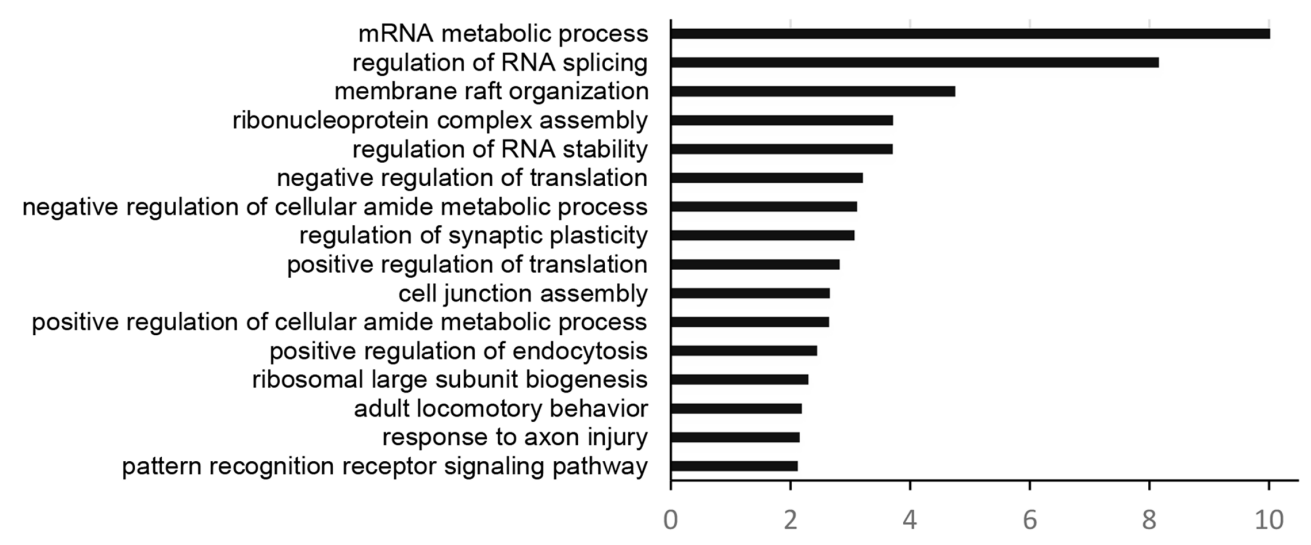

\section{Molecular Function}

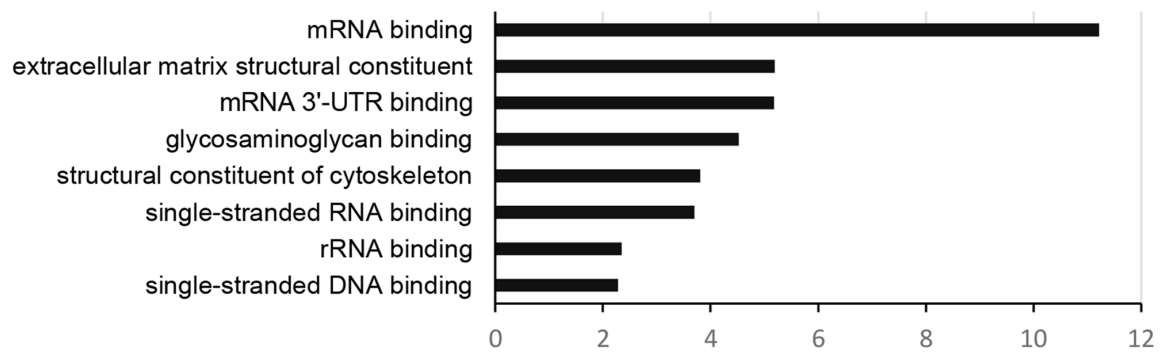

B

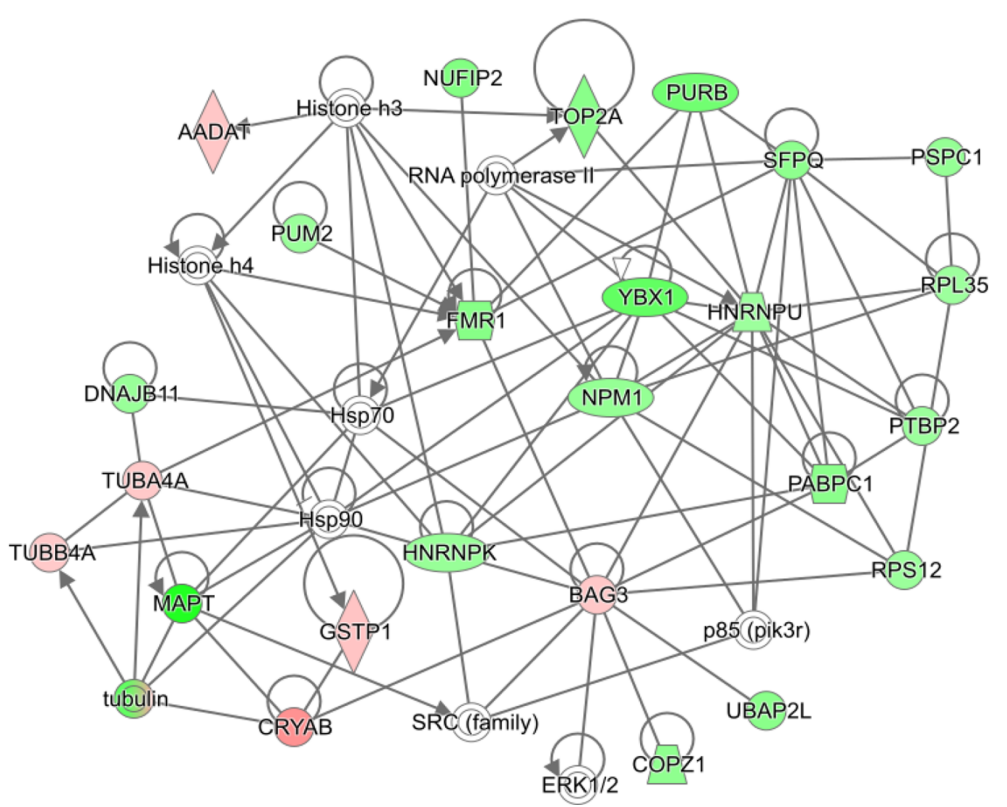

FIGURE 2 | Functional enrichment analysis and FMRP (FMR1)-associated interaction network of significantly changed proteins in aging rats. (A) The GO categories of the most regulated biological processes and molecular functions associated with significantly changed proteins in the aging group. The GO enrichment analysis (Benjamini-Hochberg $p$-Values < 0.01) was performed using ClueGO-Cytoscape platform (Bindea et al., 2009; Huntley et al., 2015). (B) The protein interaction network of significantly changed proteins centered around FMRP retrieved from IPA analysis (QIAGEN). Green color represents downregulated proteins and red color represents upregulated proteins in the aging group. The protein nodes are connected based on RNA/DNA - interactions (directional lines) or protein - protein interactions (simple lines). Autoregulation is represented by loops. The gene abbreviations are given in the text and Supplementary Table S1. 
functions $(p<0.05)$. The aging-dependent downregulation of EIF2 signaling $(\mathrm{z}$-score $=2.646)$ followed by dysregulation in 14-3-3 mediated signaling, axonal guidance signaling and cell junction signaling were found as the most significant pathways. The analysis of biological functions showed coordinated expression changes leading to an increase of RNA splicing $(\mathrm{z}$-score $=2.00)$, cell-to-cell contact $(\mathrm{z}$-score $=2.05)$, a decrease in development of neurons $(z$-score $=-2.13)$ and formation of microtubules ( $\mathrm{z}$-score $=-2.14$ ) in the aging group. The complete results obtained from GO enrichment and IPA analysis are provided as a supporting information (Supplementary Table S2).

The STRING network (STRING v10.5 database ${ }^{2}$ ) of significant proteins formed several clusters of closely related members associated with previously enriched processes. Most of the clusters were connected with each other reflecting the functional closeness of the identified machineries (Supplementary Figure S2). These include small groups of proteins involved in the formation of membrane structures and membrane vesicle trafficking (FLOT1, FLOT2, PASCIN2, BIN1, CLINT1), proteins associated with cell junctions (GJB2 GJB6, GJA1), and separate cluster of proteoglycans and proteoglycanlinked proteins (HAPLN1, VCAN, BCAN, ACAN, NCAN, TNC) linked to the group containing guanidine nucleotide binding (G) proteins (GNB4, GNG13). The enrichment of RNA metabolic activity in the significant proteomic dataset was represented by two big interconnected clusters associated with RNA processing and translation. Both clusters are linked to RNA-binding regulators involved in RNA transport and stability including FMRP, CAPRIN1, the members of Pumilio (PUM) and CUG-BP, Elav-like (CELF) family, and YTH N(6)-methyladenosine RNA-binding proteins (YTHDF).

The IPA network analysis on the individual RNA/DNA protein or protein - protein interactions of FMRP with the other significant proteins connected FMRP to nuclear FMRP interactor - NUFIP2, RNA-binding regulator - PUM2, purine rich element binding protein - PURB, splicing factor - SFPQ, BCL2 binding anthanogene - BAG3 and tubulin alpha-4A chain - TUBAA. Additionally, the network showed close association to several signaling nodes of ERK1/2 and SRC family and to general regulation of gene expression through histones and RNA polymerase (Figure 2B).

\section{DISCUSSION}

The critical role of FMRP in hippocampus was previously demonstrated in human Fragile $\mathrm{X}$ syndrome (FXR) patients and mouse FMR1 KO FXR models. A large spectrum of FXRassociated pathological changes found in mouse hippocampus include altered morphology of dendritic spines, behavioral changes, impairment of hippocampal synaptic plasticity and hippocampal-dependent memory tasks (reviewed in Bostrom et al., 2016). Furthemore, deficits in long-term memory in hippocampus and spatial learning were observed in rat models

${ }^{2}$ https://string-db.org/ of FXR (Tian et al., 2017). Many of FXR symptoms share a high degree of similarity to systemic changes and cognitive decline during normal aging which points to aging-related changes in the processes regulated by FMRP.

The lack of FMRP in mouse and rat $\mathrm{KO}$ models have been previously associated with impaired functionality of DG and hippocampal-dependent spatial memory (Franklin et al., 2014; Hébert et al., 2014; Yau et al., 2016) with both affected during normal aging in mammals (Lister and Barnes, 2009). In the current study, FMRP levels were analyzed in the DG of young (17 weeks) and aging (22 months) rats and the results were confirmed by three different methodological approaches - quantitative proteomics, immunoblotting, and immunofluorescence microscopy. The cohorts of both animal groups were first behaviorally tested for performance in a hippocampal-dependent memory task and confirmed a significant decline in spatial reference memory in aging rats. Proteomic results showed a significant reduction of FMRP levels in the samples from the aging group. Immunoblotting was performed with KO-verified antibody MAB2160, previously used for analysis of FMRP in a rat FXR model (Till et al., 2015). Several isoforms of FMRP around $70 \mathrm{kDa}$ were detected with all signals significantly decreased in the aging rats. Consistently with proteomic- and immunoblotting data, the reduction of FMRP levels in total area or individual subareas of aging DG was confirmed by immunofluorescence microscopy.

Reduction of FMRP in aging rats observed in the current study corroborates with the previous studies on changes of FMRP levels in total brain tissue from young and aging mice (Singh et al., 2007; Singh and Prasad, 2008). Interestingly, the authors found sex-related discrepancies in the level changes of FMR1 mRNA with consistent reduction of FMRP in the aging group which was attributed to interactions with specific transcription factors (Singh and Prasad, 2008). All rats used in the experiments presented herein were males and the corresponding sex-related differences in the levels of FMRP and FMR1 mRNA in the aging rat brain remain to be investigated.

In addition to FMRP the quantitative proteomic experiment allowed simultaneous detection of 153 proteins that were significantly up- or downregulated in the aging compared to young rats. Based on functional enrichment- and network analysis these proteins could be clustered into several groups implicated in the altered biological processes. The highest protein ratios were detected for proteoglycans and proteoglycanassociated proteins brevican (BCAN), neurocan (NCAN), versican (VCAN), aggrecan (ACAN), tenscin (TNC), and hyaluronan and proteoglycan link proteins (HAPLNs). These proteins are part of the extracellular brain matrix that plays a numerous roles in brain morphogenesis by controlling migration, proliferation and differentiation of neurons and facilitating molecular signaling (Oohashi et al., 2015). The experiments in $\mathrm{KO}$ mice proved that these molecules have an essential function in maintaining synaptic plasticity in the DG (Jansen et al., 2017). The proteins of membrane vesicles, lipid rafts and cell junctions formed another group significantly deregulated in the aging rats. These include Flotillins (FLOTs), Bridge integrator-1 (BIN1) or 
Protein Kinase C And Casein Kinase Substrate In Neurons 2 (PACSIN2) that were previously linked to synapse formation and function in hippocampus (Swanwick et al., 2010; Anggono et al., 2013; Monje et al., 2013; Zhang et al., 2015).

Proteins related to translation machinery and RNA processing were the most represented group among the dysregulated proteins. The synthesis of new proteins is a key element for basic cellular functions but has been also implicated more specifically in memory formation. It was shown that protein synthesis is important for synaptic remodeling underlying the longterm memory (Sutton and Schuman, 2006; Alvarez-Castelao and Schuman, 2015), involved in the consolidation phase of hippocampal-dependent learning (Rossato et al., 2007) and has a role in hippocampal synaptic plasticity (Cajigas et al., 2010). The pathway enrichment analysis from the current study revealed a significant downregulation of EIF2 signaling ( $\mathrm{z}$ score $=2.646$ ) representing a decrease in protein synthesis in DGs of the aging group. This has been also reported by previous studies on young and aging Sprague-Dawley rats (Ingvar et al., 1985; Smith et al., 1995) and represents confirmation of the proteomic results. Interestingly, the study of Wei et al. (2015) recently revealed an important function of RNA-binding regulators in decoupling of transcription and translation processes during aging in prefrontal cortex of humans and monkeys. The network analysis of significant proteins presented herein connected the clusters of mRNA translation and splicing machineries to FMRP and several other RNA-binding regulators involved in RNA transport and stability including CAPRIN1, the members of Pumilio (PUM) and CUG-BP, Elav-like (CELF) family, and YTH $\mathrm{N}(6)$-methyladenosine RNA-binding proteins (YTHDF). PUM proteins are closely related to FMRP through RNA-interactions and number of functions including DG neurogenesis and synaptic morphogenesis (Vessey et al., 2010; Zhang et al., 2017). CELF proteins has been proposed as regulators of synaptic plasticity and transmission in hippocampus (Wagnon et al., 2012).

\section{REFERENCES}

Adinolfi, S., Bagni, C., Musco, G., Gibson, T., Mazzarella, L., and Pastore, A. (1999). Dissecting FMR1, the protein responsible for fragile X syndrome, in its structural and functional domains. RNA 5, 1248-1258. doi: 10.1017/ S1355838299990647

Alvarez-Castelao, B., and Schuman, E. M. (2015). The regulation of synaptic protein turnover. J. Biol. Chem. 290, 28623-28630. doi: 10.1074/jbc.R115. 657130

Anggono, V., Koc-Schmitz, Y., Widagdo, J., Kormann, J., Quan, A., Chen, C. M., et al. (2013). PICK1 interacts with PACSIN to regulate AMPA receptor internalization and cerebellar long-term depression. Proc. Natl. Acad. Sci. U.S.A. 110, 13976-13981. doi: 10.1073/pnas.1312467110

Antar, L. N., Afroz, R., Dictenberg, J. B., Carroll, R. C., and Bassell, G. J. (2004). Metabotropic glutamate receptor activation regulates fragile $\mathrm{x}$ mental retardation protein and FMR1 mRNA localization differentially in dendrites and at synapses. J. Neurosci. 24, 2648-2655. doi: 10.1523/JNEUROSCI.009904.2004

Ascano, M. Jr., Mukherjee, N., Bandaru, P., Miller, J. B., Nusbaum, J. D., Corcoran, D. L., et al. (2012). FMRP targets distinct mRNA sequence elements

\section{CONCLUSION}

The study presented herein showed that the level of the functional synaptic regulator, FMRP, in the rat DG is significantly reduced during aging. In addition, quantitative proteomics and bioinformatics identified several dysregulated protein clusters. The results emphasized the role of FMRP and other RNAbinding regulators in the modulation of DG protein synthesis during aging that warrant further analysis. The complete list of dysregulated proteins obtained from current study is presented in the result section and supplementary material, and might provide a useful dataset for further studies on aging in the rat hippocampus.

\section{AUTHOR CONTRIBUTIONS}

Conceived and designed the experiments: RS, FS, VK, HH, JW, and GL; performing experiments: RS, MK, TS, DR, JM, DF, and JW; collected data and processed them: RS, FS, TS, JM, DF, DR, and VK; interpreted the results: RS, GL, DM, and JW; wrote paper: RS, GL, VK, and DR; revised the intellectual content: GL, VK, DM, and HH. All authors read and approved the manuscript.

\section{SUPPLEMENTARY MATERIAL}

The Supplementary Material for this article can be found online at: https://www.frontiersin.org/articles/10.3389/fnagi. 2017.00384/full\#supplementary-material

TABLE S1 | The list of proteins identified in the quantitative proteomic study and the proteins with significantly changed levels between young and aging rats. The identified protein datasets with UniProt accession numbers, MS parameters and the statistics of the animal group comparison.

TABLE S2 | The GO enrichment analysis and the identification of the most representative biological functions and canonical pathways associated with the differential protein dataset obtained from the quantitative proteomic study.

to regulate protein expression. Nature 492, 382-386. doi: 10.1038/nature 11737

Bindea, G., Mlecnik, B., Hackl, H., Charoentong, P., Tosolini, M., Kirilovsky, A., et al. (2009). ClueGO: a Cytoscape plug-in to decipher functionally grouped gene ontology and pathway annotation networks. Bioinformatics 25, 1091-1093. doi: 10.1093/bioinformatics/btp101

Bostrom, C., Yau, S. Y., Majaess, N., Vetrici, M., Gil-Mohapel, J., and Christie, B. R. (2016). Hippocampal dysfunction and cognitive impairment in Fragile-X Syndrome. Neurosci. Biobehav. Rev. 68, 563-574. doi: 10.1016/j.neubiorev.2016. 06.033

Bott, J. B., Muller, M. A., Jackson, J., Aubert, J., Cassel, J. C., Mathis, C., et al. (2016). Spatial reference memory is associated with modulation of theta-gamma coupling in the dentate gyrus. Cereb. Cortex 26, 3744-3753. doi: 10.1093/cercor/ bhv177

Cajigas, I. J., Will, T., and Schuman, E. M. (2010). Protein homeostasis and synaptic plasticity. EMBO J. 29, 2746-2752. doi: 10.1038/emboj.2010.173

Eberhart, D. E., Malter, H. E., Feng, Y., and Warren, S. T. (1996). The fragile $\mathrm{X}$ mental retardation protein is a ribonucleoprotein containing both nuclear localization and nuclear export signals. Hum. Mol. Genet. 5, 1083-1091. doi: $10.1093 / \mathrm{hmg} / 5.8 .1083$ 
Edbauer, D., Neilson, J. R., Foster, K. A., Wang, C. F., Seeburg, D. P., Batterton, M. N., et al. (2010). Regulation of synaptic structure and function by FMRPassociated microRNAs miR-125b and miR-132. Neuron 65, 373-384. doi: 10 1016/j.neuron.2010.01.005

Feng, Y., Gutekunst, C. A., Eberhart, D. E., Yi, H., Warren, S. T., and Hersch, S. M. (1997). Fragile X mental retardation protein: nucleocytoplasmic shuttling and association with somatodendritic ribosomes. J. Neurosci. 17, 1539-1547.

Franklin, A. V., King, M. K., Palomo, V., Martinez, A., McMahon, L. L., and Jope, R. S. (2014). Glycogen synthase kinase-3 inhibitors reverse deficits in longterm potentiation and cognition in fragile X mice. Biol. Psychiatry 75, 198-206. doi: 10.1016/j.biopsych.2013.08.003

Gilar, M., Olivova, P., Daly, A. E., and Gebler, J. C. (2005). Two-dimensional separation of peptides using RP-RP-HPLC system with different $\mathrm{pH}$ in first and second separation dimensions. J. Sep. Sci. 28, 1694-1703. doi: 10.1002/jssc. 200500116

Greenough, W. T., Klintsova, A. Y., Irwin, S. A., Galvez, R., Bates, K. E., and Weiler, I. J. (2001). Synaptic regulation of protein synthesis and the fragile $\mathrm{X}$ protein. Proc. Natl. Acad. Sci. U.S.A. 98, 7101-7106. doi: 10.1073/pnas.141145998

Hébert, B., Pietropaolo, S., Meme, S., Laudier, B., Laugeray, A., Doisne, N., et al. (2014). Rescue of fragile X syndrome phenotypes in Fmr1 KO mice by a BKCa channel opener molecule. Orphanet J. Rare Dis. 9, 124. doi: 10.1186/s13023014-0124-6

Huntley, R. P., Sawford, T., Mutowo-Meullenet, P., Shypitsyna, A., Bonilla, C., Martin, M. J., et al. (2015). The GOA database: gene Ontology annotation updates for 2015. Nucleic Acids Res. 43, D1057-D1063. doi: 10.1093/nar/ gku1113

Ingvar, M. C., Maeder, P., Sokoloff, L., and Smith, C. B. (1985). Effects of ageing on local rates of cerebral protein synthesis in Sprague-Dawley rats. Brain 108(Pt 1), 155-170. doi: 10.1093/brain/108.1.155

Irwin, S. A., Christmon, C. A., Grossman, A. W., Galvez, R., Kim, S. H., DeGrush, B. J., et al. (2005). Fragile X mental retardation protein levels increase following complex environment exposure in rat brain regions undergoing active synaptogenesis. Neurobiol. Learn. Mem. 83, 180-187. doi: 10.1016/j.nlm. 2004.11.004

Jansen, S., Gottschling, C., Faissner, A., and Manahan-Vaughan, D. (2017). Intrinsic cellular and molecular properties of in vivo hippocampal synaptic plasticity are altered in the absence of key synaptic matrix molecules. Hippocampus 27 , 920-933. doi: 10.1002/hipo.22742

Jin, P., and Warren, S. T. (2000). Understanding the molecular basis of fragile $\mathrm{X}$ syndrome. Hum. Mol. Genet. 9, 901-908. doi: 10.1093/hmg/9.6.901

Jonas, P., and Lisman, J. (2014). Structure, function, and plasticity of hippocampal dentate gyrus microcircuits. Front. Neural Circuits 8:107. doi: 10.3389/fncir. 2014.00107

Kalidas, S., and Smith, D. P. (2003). Functional genomics, fragile X syndrome, and RNA interference. Arch. Neurol. 60, 1197-1200. doi: 10.1001/archneur.60. 9.1197

Lai, J. K., Doering, L. C., and Foster, J. A. (2016). Developmental expression of the neuroligins and neurexins in fragile X mice. J. Comp. Neurol. 524, 807-828. doi: $10.1002 /$ cne. 23868

Li, Z., Zhang, Y., Ku, L., Wilkinson, K. D., Warren, S. T., and Feng, Y. (2001). The fragile $\mathrm{X}$ mental retardation protein inhibits translation via interacting with mRNA. Nucleic Acids Res. 29, 2276-2283. doi: 10.1093/nar/29.11.2276

Lister, J. P., and Barnes, C. A. (2009). Neurobiological changes in the hippocampus during normative aging. Arch. Neurol. 66, 829-833. doi: 10.1001/archneurol. 2009.125

Monje, F. J., Divisch, I., Demit, M., Lubec, G., and Pollak, D. D. (2013). Flotillin-1 is an evolutionary-conserved memory-related protein up-regulated in implicit and explicit learning paradigms. Ann. Med. 45, 301-307. doi: 10. 3109/07853890.2013.770637

Nakamoto, M., Nalavadi, V., Epstein, M. P., Narayanan, U., Bassell, G. J., and Warren, S. T. (2007). Fragile X mental retardation protein deficiency leads to excessive mGluR5-dependent internalization of AMPA receptors. Proc. Natl. Acad. Sci. U.S.A. 104, 15537-15542. doi: 10.1073/pnas.0707484104

Oohashi, T., Edamatsu, M., Bekku, Y., and Carulli, D. (2015). The hyaluronan and proteoglycan link proteins: organizers of the brain extracellular matrix and key molecules for neuronal function and plasticity. Exp. Neurol. 274, 134-144. doi: 10.1016/j.expneurol.2015.09.010
Ouwenga, R. L., and Dougherty, J. (2015). Fmrp targets or not: long, highly brainexpressed genes tend to be implicated in autism and brain disorders. Mol. Autism 6, 16. doi: 10.1186/s13229-015-0008-1

Rappsilber, J., Mann, M., and Ishihama, Y. (2007). Protocol for micro-purification, enrichment, pre-fractionation and storage of peptides for proteomics using StageTips. Nat. Protoc. 2, 1896-1906. doi: 10.1038/nprot.2007.261

Rossato, J. I., Bevilaqua, L. R., Myskiw, J. C., Medina, J. H., Izquierdo, I., and Cammarota, M. (2007). On the role of hippocampal protein synthesis in the consolidation and reconsolidation of object recognition memory. Learn. Mem. 14, 36-46. doi: 10.1101/lm.422607

Scharkowski, F., Frotscher, M., Lutz, D., Korte, M., and Michaelsen-Preusse, K. (2017). Altered connectivity and synapse maturation of the hippocampal mossy fiber pathway in a mouse model of the fragile $\mathrm{X}$ syndrome. Cereb. Cortex doi: 10.1093/cercor/bhw408 [Epub ahead of print].

Shanmugasundaram, B., Aher, Y. D., Aradska, J., Ilic, M., Daba Feyissa, D., Kalaba, P., et al. (2017). R-Modafinil exerts weak effects on spatial memory acquisition and dentate gyrus synaptic plasticity. PLOS ONE 12:e0179675. doi: 10.1371/journal.pone.0179675

Singh, K., Gaur, P., and Prasad, S. (2007). Fragile x mental retardation (Fmr-1) gene expression is down regulated in brain of mice during aging. Mol. Biol. Rep. 34, 173-181. doi: 10.1007/s11033-006-9032-8

Singh, K., and Prasad, S. (2008). Differential expression of Fmr-1 mRNA and FMRP in female mice brain during aging. Mol. Biol. Rep. 35, 677-684. doi: 10.1007/s11033-007-9140-0

Smidak, R., Aradska, J., Kirchberger, S., Distel, M., Sialana, F. J., Wackerlig, J. et al. (2016). A detailed proteomic profiling of plasma membrane from zebrafish brain. Proteomics Clin. Appl. 10, 1264-1268. doi: 10.1002/prca.201600081

Smith, C. B., Sun, Y., and Sokoloff, L. (1995). Effects of aging on regional rates of cerebral protein synthesis in the Sprague-Dawley rat: examination of the influence of recycling of amino acids derived from protein degradation into the precursor pool. Neurochem. Int. 27, 407-416. doi: 10.1016/0197-0186(95) $00022-Z$

Sutton, M. A., and Schuman, E. M. (2006). Dendritic protein synthesis, synaptic plasticity, and memory. Cell 127, 49-58. doi: 10.1016/j.cell.2006.09.014

Swanwick, C. C., Shapiro, M. E., Vicini, S., and Wenthold, R. J. (2010). Flotillin-1 promotes formation of glutamatergic synapses in hippocampal neurons. Dev. Neurobiol. 70, 875-883. doi: 10.1002/dneu.20828

Taha, M. S., Nouri, K., Milroy, L. G., Moll, J. M., Herrmann, C., Brunsveld, L., et al. (2014). Subcellular fractionation and localization studies reveal a direct interaction of the fragile $\mathrm{X}$ mental retardation protein (FMRP) with nucleolin. PLOS ONE 9:e91465. doi: 10.1371/journal.pone.0091465

Tamanini, F., Willemsen, R., van Unen, L., Bontekoe, C., Galjaard, H., Oostra, B. A., et al. (1997). Differential expression of FMR1, FXR1 and FXR2 proteins in human brain and testis. Hum. Mol. Genet. 6, 1315-1322. doi: 10.1093/hmg/ 6.8 .1315

Tang, B., Wang, T., Wan, H., Han, L., Qin, X., Zhang, Y., et al. (2015). Fmrl deficiency promotes age-dependent alterations in the cortical synaptic proteome. Proc. Natl. Acad. Sci. U.S.A. 112, E4697-E4706. doi: 10.1073/pnas. 1502258112

Tian, Y., Yang, C., Shang, S., Cai, Y., Deng, X., Zhang, J., et al. (2017). Loss of FMRP impaired hippocampal long-term plasticity and spatial learning in rats. Front. Mol. Neurosci. 10:269. doi: 10.3389/fnmol.2017.00269

Till, S. M., Asiminas, A., Jackson, A. D., Katsanevaki, D., Barnes, S. A., Osterweil, E. K., et al. (2015). Conserved hippocampal cellular pathophysiology but distinct behavioural deficits in a new rat model of FXS. Hum. Mol. Genet. 24, 5977-5984. doi: 10.1093/hmg/ddv299

Till, S. M., Wijetunge, L. S., Seidel, V. G., Harlow, E., Wright, A. K., Bagni, C., et al. (2012). Altered maturation of the primary somatosensory cortex in a mouse model of fragile X syndrome. Hum. Mol. Genet. 21, 2143-2156. doi: $10.1093 / \mathrm{hmg} / \mathrm{dds} 030$

Valverde, R., Pozdnyakova, I., Kajander, T., Venkatraman, J., and Regan, L. (2007). Fragile X mental retardation syndrome: structure of the KH1-KH2 domains of fragile X mental retardation protein. Structure 15, 1090-1098. doi: 10.1016/j.str. 2007.06.022

Vessey, J. P., Schoderboeck, L., Gingl, E., Luzi, E., Riefler, J., Di Leva, F., et al. (2010). Mammalian Pumilio 2 regulates dendrite morphogenesis and synaptic function. Proc. Natl. Acad. Sci. U.S.A. 107, 3222-3227. doi: 10.1073/pnas.09071 28107 
Wagnon, J. L., Briese, M., Sun, W., Mahaffey, C. L., Curk, T., Rot, G., et al. (2012). CELF4 regulates translation and local abundance of a vast set of mRNAs, including genes associated with regulation of synaptic function. PLOS Genet. 8:e1003067. doi: 10.1371/journal.pgen.1003067

Wang, T., Bray, S. M., and Warren, S. T. (2012). New perspectives on the biology of fragile X syndrome. Curr. Opin. Genet. Dev. 22, 256-263. doi: 10.1016/j.gde. 2012.02.002

Wei, Y. N., Hu, H. Y., Xie, G. C., Fu, N., Ning, Z. B., Zeng, R., et al. (2015). Transcript and protein expression decoupling reveals RNA binding proteins and miRNAs as potential modulators of human aging. Genome Biol. 16, 41. doi: 10.1186/s13059-015-0608-2

Weiler, I. J., Spangler, C. C., Klintsova, A. Y., Grossman, A. W., Kim, S. H., BertainaAnglade, V., et al. (2004). Fragile X mental retardation protein is necessary for neurotransmitter-activated protein translation at synapses. Proc. Natl. Acad. Sci. U.S.A. 101, 17504-17509. doi: 10.1073/pnas.0407533101

Wisniewski, J. R., Zougman, A., Nagaraj, N., and Mann, M. (2009). Universal sample preparation method for proteome analysis. Nat. Methods 6, 359-362. doi: 10.1038/nmeth.1322

Yau, S. Y., Bostrom, C. A., Chiu, J., Fontaine, C. J., Sawchuk, S., Meconi, A., et al. (2016). Impaired bidirectional NMDA receptor dependent synaptic plasticity in the dentate gyrus of adult female Fmr1 heterozygous knockout mice. Neurobiol. Dis. 96, 261-270. doi: 10.1016/j.nbd.2016.09.012

Zalfa, F., Eleuteri, B., Dickson, K. S., Mercaldo, V., De Rubeis, S., di Penta, A., et al. (2007). A new function for the fragile $\mathrm{X}$ mental retardation protein in regulation of PSD-95 mRNA stability. Nat. Neurosci. 10, 578-587. doi: 10.1038/nn 1893

Zhang, M., Chen, D., Xia, J., Han, W., Cui, X., Neuenkirchen, N., et al. (2017). Posttranscriptional regulation of mouse neurogenesis by Pumilio proteins. Genes Dev. doi: 10.1101/gad.298752.117 [Epub ahead of print].

Zhang, X., Yu, J. T., Li, J., Wang, C., Tan, L., Liu, B., et al. (2015). Bridging integrator 1 (BIN1) genotype effects on working memory, hippocampal volume, and functional connectivity in young healthy individuals. Neuropsychopharmacology 40, 1794-1803. doi: 10.1038/npp.2015.30

Zimmer, S. E., Doll, S. G., Garcia, A. D. R., and Akins, M. R. (2017). Splice formdependent regulation of axonal arbor complexity by FMRP. Dev. Neurobiol. 77, 738-752. doi: 10.1002/dneu.22453

Conflict of Interest Statement: The authors declare that the research was conducted in the absence of any commercial or financial relationships that could be construed as a potential conflict of interest.

Copyright (c) 2017 Smidak, Sialana, Kristofova, Stojanovic, Rajcic, Malikovic, Feyissa, Korz, Hoeger, Wackerlig, Mechtcheriakova and Lubec. This is an open-access article distributed under the terms of the Creative Commons Attribution License (CC BY). The use, distribution or reproduction in other forums is permitted, provided the original author(s) or licensor are credited and that the original publication in this journal is cited, in accordance with accepted academic practice. No use, distribution or reproduction is permitted which does not comply with these terms. 